\title{
COVID-19 and liver transplantation
}

\author{
Tommaso Di Maira ${ }^{1}$ and Marina Berenguer ${ }^{2 凶}$
}

The effect of coronavirus disease 2019 (COVID-19) on liver transplantation programmes and recipients is still not completely understood but overall involves the risk of donor-derived transmission, the reliability of diagnostic tests, health-care resource utilization and the effect of immunosuppression. This Comment reviews the effect of COVID-19 on liver transplantation and summarizes recommendations for donor and recipient management.

Liver transplantation (LT) programmes worldwide have been deeply affected by coronavirus disease 2019 (COVID-19), which is caused by severe acute respiratory syndrome coronavirus 2 (SARS-CoV-2). Solid organ transplantation activity has decreased even in regions where COVID-19 prevalence was low, which suggests a global and nationwide effect beyond the local COVID-19 prevalence (Supplementary Fig. 1).

Given the speed of the SARS-CoV-2 outbreak, no scientific evidence regarding LT is available to guide medical policymakers during this period of readjustment. Yet, given the potential negative consequences, multiple organizations have released recommendations stating that all potential organs from deceased donors with SARS-CoV-2 infection should be deferred, including those from otherwise medically eligible donors with mild or asymptomatic SARS-CoV-2 infection. Most programmes recommend screening deceased donors to prevent the inadvertent transplantation of organs from a SARS-CoV-2-infected donor ${ }^{1}$. However, owing to a scarcity of deceased donor organs and high risk of waitlist mortality, some advocate the utilization of COVID-19-positive liver donors in selected cases. The rationale is the low (albeit not null) proportion of viraemic cases and no recognized SARS-CoV-2 transfusion transmissions, as well as the absence of evidence of SARS-CoV-2 infection in liver in the very limited number of autopsy studies ${ }^{2}$.

Notably, although the potential for SARS-CoV-2 transmission by non-lung organ donation is unclear, a case of COVID-19-associated hepatitis in the recipient of a living donor liver allograft, whose donor subsequently tested positive for COVID-19, has been described (Supplementary Table 1). Furthermore, the potential negative consequences of COVID-19-positive donations not only to the recipients but also to the transplant team, organ procurement organization and associated hospital workforce community, particularly in the absence of effective therapy, argues against this strategy. In this scenario, donor screening using both clinical and laboratory approaches remains an important consideration.
Most societies recommend a thorough epidemiological and clinical history screening alongside donor testing for COVID-19 (nucleic acid testing (NAT) of nasopharyngeal specimens of donor and recipient) (Supplementary Box 1) ${ }^{1,3}$. Donor testing should be executed preferentially by bronchoalveolar lavage in deceased donors or by nasopharyngeal swab in living donors $^{1}$. As no test is $100 \%$ sensitive or specific ${ }^{4}$, risk stratification by epidemiological and clinical screening as recommended by the AST (see Related links) should be considered.

In countries with a high incidence of COVID-19, a positive epidemiological screening (that is, residence in or travel to a high-risk area, or contact with a suspected or confirmed case) can be assumed positive. Positive clinical screening includes the onset of any of a set of symptoms 21 days before evaluation (FIG. 1; Supplementary Box 1). Donors who are positive for both the epidemiological and clinical screenings are considered high risk, while one or no positive screening denote intermediate or low risk, respectively. The organs of donors who test positive for COVID-19 by NAT or who are considered high risk without available NAT should not be used for transplantation. In the absence of available testing, only four professional societies consider it acceptable that organs be procured according to epidemiological and clinical risk stratification ${ }^{1}$ (FIG. 1; Supplementary Box 1).

Liver grafts recovered from COVID-19-positive deceased donors 21-28 days after symptom resolution and two negative swabs $24 \mathrm{~h}$ apart are considered safe for transplantation. High-risk donors with clinical symptoms within 28 days before organ procurement should not be considered even if they test negative (Supplementary Box 1$)^{3}$.

For living donors, most societies recommend testing donors at least once within 24-72 days before transplantation. Moreover, 14-21 days' self or hospital-based quarantining has been recommended together with preventive transmission counselling. COVID-19-positive or high-risk living donors should postpone donation until at least 21-28 days after symptom resolution 


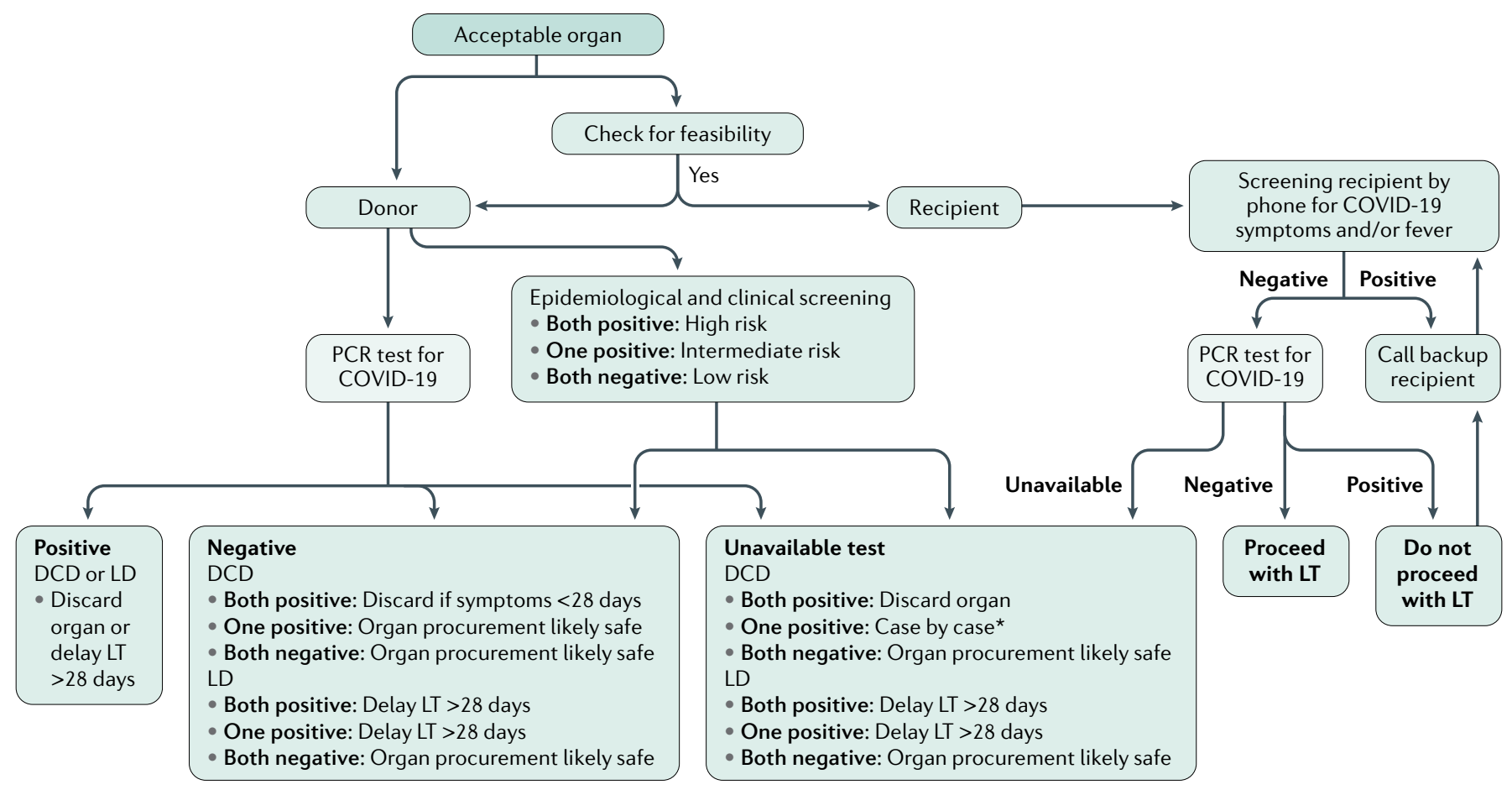

Fig. 1 | Approach to liver transplantation during COVID-19. Epidemiological screening includes any of the following: travel or residence in a high-risk area, contact with a suspected case of coronavirus disease 2019 (COVID-19) $\leq 21$ days before, or contact with a confirmed case of COVID-19 $\leq 28$ days before. Clinical screening includes onset of any of the following symptoms 21 days before evaluation: fever $>38^{\circ} \mathrm{C}$; malaise or flu-like symptoms; new cough; shortness of breath; unexplained abdominal pain, nausea or diarrhoea; or loss of sense of taste or smell. *Liver transplantation (LT) decision should be made with caution and at least 28 days after presumptive diagnosis of COVID-19. Figure is based on the main international transplant societies' recommendations (such as AST and AASLD). DCD, deceased donor; LD, living donor. For further details, see Supplementary Box 1.

and until two negative PCR tests have been observed (Supplementary Box 1$)^{3}$. The recipient should undergo a real-time PCR test within $24 \mathrm{~h}$ of transplantation. Recipients with clinical suspicion or active SARS-CoV-2 infection should have transplantation deferred until 28 days after symptom resolution and after two negative tests at least $24 \mathrm{~h}$ apart ${ }^{1}$.

The decision to pause a life-saving procedure such as LT becomes increasingly difficult as waitlist mortality increases. To optimize transplant activity and limit collateral damage, a system based on risk tolerance, hospital capacity and degree of virus activity in the jurisdiction has been proposed. This phased approach would mean that urgent transplantations would proceed first, while 'elective' cases could be phased in later based on Model for End-Stage Liver Disease (MELD) score, risk of drop-out (particularly from liver cancer progression) and fulminant hepatic failure ${ }^{3}$. Living and deceased donor LTs would be performed preferentially in clinically unstable patients according to intensive care unit capacities and facilities ${ }^{3}$. Most professional societies recommend the temporary cessation of elective living donor and non-urgent deceased donor liver transplantation during the pandemic ${ }^{1}$ (Supplementary Fig. 2).

Liver injury has been widely reported in COVID-19 (REF. ${ }^{5}$ ), particularly in patients with severe disease. Pre-existing liver disease ${ }^{6}$, comorbidities, drug toxicity or the complex systemic inflammatory response syndrome associated with the infection might also explain liver injury ${ }^{5}$.

On the basis of data from other viruses, including SARS-CoV, one would argue that immunosuppressed patients with COVID-19 are at increased risk of higher viral loads, severe clinical manifestations and prolonged shedding ${ }^{3}$. Yet, preliminary data in LT suggest that COVID-19 ranges from mild to severe, with similar or lower incidence than the general population (Supplementary Table 1). Unfortunately, data on COVID-19 in solid organ transplantation, and particularly in LT, are based on short and uncontrolled series with conflicting results. The incidence of COVID-19 in a large Italian LT survey $(n=640)$ was only $1.25 \%$, and most patients (75\%) had mild disease ${ }^{7}$. Conversely, a report showed worse outcome in solid organ transplantation recipients in New York City, USA ${ }^{8}$. Long-term $\mathrm{LT}$ recipients seem to be more prone to severe disease than short-term LT patients, suggesting that immunosuppression per se does not increase the risk of severe COVID-19, and that alternatively, the presence of metabolic-related comorbidities typically observed in long-term recipients is responsible for the increased risk of severe COVID-19 in this population ${ }^{9}$. Prolonged shedding is also relevant in terms of viral spreading and the need for long quarantine periods. Although no convincing data have been published, case reports have 
described positive PCR tests lasting up to 36 days from clinical onset (Supplementary Table 1).

According to the WHO, there is no specific treatment or vaccine for COVID-19. Therefore, treatment of LT recipients should be based on the principles of acute respiratory distress syndrome therapy. Although controversial, data suggest that an altered immune response might be the main driver of pulmonary injury, thereby indicating a potential protective role of immunosuppression ${ }^{10}$. Society recommendations are based on the general approach to transplant recipients with active infection, whereby minimization of immunosuppression is typically beneficial and the trade-off between the benefit and risk of graft rejection must be considered. Overall, most societies advise against immunosuppression modifications in patients with mild COVID-19, whereas reduction or discontinuation of antiproliferative agents and lymphocyte-depleting therapies has been suggested in special circumstances such as severe lymphopenia, worsening pneumonia or bacterial or fungal superinfection (for example, by the AASLD; see Related links). Furthermore, experimental COVID-19 treatments might interact with immunosuppressive drugs, leading to an increased risk of graft rejection or toxicity. More data are needed on LT and COVID-19 to inform treatment decisions.

1. Ritschl, P. V. et al. Solid organ transplantation programs facing lack of empiric evidence in the COVID-19 pandemic: A By-proxy Society Recommendation Consensus approach. Am. J. Transplant https://doi.org/10.1111/ajt.15933 (2020).

2. Kates, O. S., Fisher, C. E., Rakita, R. M., Reyes, J. D. \& Limaye, A. P. Use of SARS-CoV-2 infected deceased organ donors: Should we always "just say no?". Am. J. Transplant. https://doi.org/10.1111/ ajt. 16000 (2020).

3. Kumar, D. et al. COVID-19: A global transplant perspective on successfully navigating a pandemic. Am. J. Transplant. https:// doi.org/10.1111/ajt.15876 (2020).

4. Fang, Y. et al. Sensitivity of chest CT for COVID-19: comparison to RT-PCR. Radiology https://doi.org/10.1148/radiol.2020200432 (2020).

5. Kukla, M. et al. COVID-19, MERS and SARS with concomitant liver injury - systematic review of the existing literature. J. Clin. Med. 9 1420 (2020).

6. Singh S. \& Khan A Clinical characteristics and outcomes of COVID-19 among patients with pre-existing liver disease in United States: a multi-center research network study. Gastroenterology https://doi.org/10.1053/j.gastro.2020.04.064 (2020).

7. Donato, M. F., Invernizzi, F., Lampertico, P. \& Rossi, G. Health status of patients who underwent liver transplantation during the coronavirus outbreak at a large center in Milan, Italy. Clin. Gastroenterol. Hepatol. https://doi.org/10.1016/j.cgh.2020.04.041 (2020).

8. Pereira, M. R. et al. COVID-19 in solid organ transplant recipients: Initial report from the US epicenter. Am. J. Transplant. https://doi. org/10.1111/ajt. 15941 (2020).

9. Bhoori, S., Rossi, R. E., Citterio, D. \& Mazzaferro, V. COVID-19 in long-term liver transplant patients: preliminary experience from an Italian transplant centre in Lombardy. Lancet Gastroenterol. Hepatol. 5, 532-533 (2020).

10. Mehta, P. et al. COVID-19: consider cytokine storm syndromes and immunosuppression. Lancet 395, 1033-1034 (2020).

\section{Competing interests}

The authors declare no competing interests.

Supplementary information

Supplementary information is available for this paper at https://doi.org/ 10.1038/s41575-020-0347-z.

\section{RELATED LINKS}

American Association for the study of Liver Disease (AASLD): https://www.

aasld.org/about-aasld/covid-19-and-liver

American Society of Transplantation (AST): https://www.myast.org/

covid-19-information 